\title{
The Antiinflammatory Action and Pharmacokinetics of a Novel Glucosamine-based Di-Peptide Aminosugar
}

Mohammad H. Gilzad-Kohan, Kamaljit Kaur and Fakhreddin Jamali

Faculty of Pharmacy and Pharmaceutical Sciences, University of Alberta, Edmonton, Alberta, Canada

Received, April 19, 2013; Revised, June 14, 2013; Accepted, June 17, 2013; Published, June 20, 2013.

\begin{abstract}
Purpose. We have previously shown favorable in vitro gut permeability for three novel dipeptide esters of glucosamine $(\mathrm{GlcN})$ likely facilitated by the peptide transporter 1 (PepT1). Herein, we report the development of a novel assay for the determination of bioavailability of the peptide ester of interest, the anti-inflammatory properties of a glycine-valine ester derivative of GlcN (GVG) as well as its pharmacokinetics under healthy and inflammatory conditions. Methods. A pre-column derivatization (with 9fluorenylmethoxycarbonyl) HPLC assay was developed to study bioavailability of GVG, GlcN or cleaved GlcN in the rats that were cannulated in their right jugular vein for blood collection. The compounds of interest were orally administered to both healthy and arthritic rats. Serial blood samples and urine were collected and assayed for the compounds. The stability of the GVG was also tested after incubation with the rat feces. Efficacy of GVG was tested in inflamed rats (injection of $0.2 \mathrm{~mL}$ of Mycobacterium butyricum in squalene) following GVG (20 and $30 \mathrm{mg} / \mathrm{kg} /$ day GlcN equivalent) or GlcN (20 and $90 \mathrm{mg} / \mathrm{kg}$ /day) administration. Arthritis index was calculated at the end of the experiment. Results. The assay was linear (ranged between $0.05-20 \mu \mathrm{g} / \mathrm{mL}$ ) and reproducible (intra- and inter-day $<10 \%$ ). Among the tested compounds, only GVG showed a significantly higher plasma concentrations and urinary excretion than GlcN ( $\approx 3$-fold increase). GVG showed a favorable stability in the rat feces. Adjuvant arthritis was completely prevented with doses greater than $20 \mathrm{mg} / \mathrm{kg} / \mathrm{day}$ with GVG being 3-fold more potent than GlcN. Conclusion. The examined glycine-valine-GlcN di-peptide aminosugar is a potent anti-inflammatory compound due to its favorable properties to deliver GlcN into the systemic circulation.
\end{abstract}

This article is open to POST-PUBLICATION REVIEW. Registered readers (see "For Readers") may comment by clicking on ABSTRACT on the issue's contents page.

\section{INTRODUCTION}

Glucosamine (GlcN) is a naturally occurring amino-sugar with anti-inflammatory properties (1$3)$. However, the oral bioavailability of GlcN is limited and, since, at least outside Europe, it is not a regulated compound, the quality of the marketed products and also those used in clinical trials are questionable (4). The latter issues have been suggested to be, at least in part, behind the controversy in the effectiveness of GlcN. Nevertheless, GlcN is vastly used in the treatment of osteoarthritis (OA) (5).

One approach for improving the oral bioavailability of poorly absorbed polar drug such as GlcN is to synthesize prodrugs that facilitate their translocation across the intestinal wall by the di/tripeptide oligopeptide transporter (PepT1). Recently, we have designed and synthesized several mono- and di-peptide GlcN ester and amide derivatives (6). Among the tested prodrugs, an ester derivative, Gly-Val-COO-GlcN (GVG) showed increased in vitro permeability through everted rat gut, which was, at least in part, efficiently mediated by PepT1. GVG was relatively stable in the gut, readily crossed the gut wall in vitro and was rapidly hydrolyzed to yield the parent drug $(\mathrm{GlcN})$ after incubation with liver homogenates; therefore, it appeared to be a promising candidate for further investigation.

The first aim of this study was to investigate the in vivo bioavailability of three di-peptide esters of GlcN, GVG, phenylalanine-phenylalanine-COOGlcN (FFG), and valine-glycine-COO-GlcN

Corresponding Author: F. Jamali, Faculty of Pharmacy and Pharmaceutical Sciences, University of Alberta, Edmonton, Alberta, Canada; Email: fjamali@ualberta.ca 
(VGG), relative to GlcN. For this purpose, we developed a sensitive HPLC assay for simultaneous measurement of $\mathrm{GlcN}$ and its ester derivative, GVG, based on fluorescence detection. Both GlcN and GVG lack chromophores; hence, they were derivatized with 9-fluorenylmethoxycarbonyl (Fmoc), a highly fluorescent compound $(7,8)$.

The second aim of this study was to assess GVG stability in the rat feces. It has been previously shown that GlcN can be degraded by feces, which in turn, causes reduced bioavailability (9).

The third aim of this study was to explore the efficacy of GVG in prevention of experimental inflammation in a rat model of adjuvant arthritis (AA), which has been used for decades to test antirhematoid drugs (10). There are several studies indicating that GlcN at high doses, which is associated with high plasma concentrations, is effective in prevention and controlling inflammatory conditions including AA $(1,2)$. We, hence, hypothesized that a GlcN prodrug (e.g. GVG) with a relatively high bioavailability is a novel drug suitable for the treatment of inflammatory conditions such as AA. The new GlcN derivative can produce therapeutic GlcN levels with smaller daily doses compared to GlcN. This is expected to improve patient's compliance as the available product are excessively large; a typical $500 \mathrm{mg}$ tablet of GlcN weighs $1.4 \mathrm{~g}$.

Finally, we investigate the possible effect of inflammation on the pharmacokinetic of GVG. Inflammation is known to cause alteration on the expression of several transporters (11). It has been reported that Dextran sulfate sodium-induced rat colitis does not alter PepT1 substrate bioavailability despite certain modifications in the mRNA expression (12).

\section{MATERIALS AND METHODS}

\section{Chemicals}

The examined dipeptides (GVG, FFG and VGG) were synthesized in our lab based on a previously described method (6). D-(+)-GlcN, 9fluorenylmethoxycarbonyl chloride (Fmoc-Cl), mannosamine $\mathrm{HCl}$, 1-aminoadmantane $\mathrm{HCl}$ (amantadine), boric acid, sodium hydroxide, lactic dehydrogenase, aspergillus nitrate reductase, FAD, NADPH, tris base, tris hydrochloride, sodium nitrite, sodium nitrate, pyruvic acid, phosphoric acid, naphthalene ethylene diamine, and sulphanilamide were purchased from SigmaAldrich (St. Louis, MO, USA). Mycobacterium butyricum was purchased from Difco (Detroit, MI, USA). Squalene was purchased from Kodak (Rochester, NY, USA). HPLC grade acetonitrile and water were purchased from Caledon Laboratories Ltd, (ON, Canada). All other chemicals and solvents were commercial products of analytical or HPLC grades.

\section{Animals}

The experimental protocol was approved by the University of Alberta Animal Care Committee. Male Sprague-Dawley rats (250-280 g) were acclimatized in a temperature-controlled room with a 12:12 $\mathrm{h}$ dark/light cycle and fed with standard rodent chow food containing crude protein $23 \%$, crude fat $4.5 \%$, crude fiber $6 \%$, ash $8 \%$, and mineral $2.5 \%$.

\section{Simultaneous HPLC assay for GIcN and GVG}

Plasma concentration and/or urinary excretion of GlcN was determined and used as a measure of bioavailability for all tested compounds. In addition, for GVG only, the intact pro-drug was measured to test its presence in various biological samples. The HPLC system consisted of a Shimadzu Prominence HPLC system (Mandel Scientific, Guelph, ON Canada) equipped with a RF-10AxL fluorescence detector, a LC-20AT pump, a DGU-20A5 degasser, a SIL-20A autosampler, a CTO-20AC column oven, a CBM-20A communication bus module and a Phenomenex $\mathrm{C} 18$ $(100 \mathrm{~mm} \times 4.6 \mathrm{~mm}$, id $3 \mu \mathrm{m})$ reversed phase column guarded with a security guard cartridge $\mathrm{C} 18$ (4 mm x3 mm) column. The gradient mobile phase consisted of $0.1 \%$ acetic acid in HPLC-grade water (A) and acetonitrile (B) which was run at 1 $\mathrm{mL} / \mathrm{min}$; the gradient system commenced and continued for $17 \mathrm{~min}$ with $24 \%$ B followed by an increase of $\mathrm{B}$ to $90 \%$ in $1 \mathrm{~min}$. The composition was maintained for $10 \mathrm{~min}$ and decreased back to $24 \%$ B in $1 \mathrm{~min}$. Column oven temperature was set at $37^{\circ} \mathrm{C}$. The detection was carried out at excitation and emission wavelength of $256 \mathrm{~nm}$ and $315 \mathrm{~nm}$, respectively. The sample run time was $38 \mathrm{~min}$. The peak height was used for all calculations.

Adequate amount of GlcN.HCl or GVG.HCl was dissolved in $10 \mathrm{~mL}$ water to yield a $1 \mathrm{mg} / \mathrm{mL}$ (based on free base) stock solution of each 
compound. The stock solutions were further diluted with water to give standard solutions containing $0.1,1,10,20$ and $40 \mu \mathrm{g} / \mathrm{mL}$. Aliquots of $0.1 \mathrm{~mL}$ plasma were spiked with the standard solutions to yield the calibration solutions containing $0.05,0.5$, 5,10 and $20 \mu \mathrm{g} / \mathrm{mL}$ of each compound. The standard curves were constructed by plotting GlcN:IS or GVG:IS peak height ratio versus the added concentration of GlcN or GVG. A $30 \mu \mathrm{g} / \mathrm{mL}$ mannosamine $\mathrm{HCl}$ (IS) solution was used as the internal standard (IS). Fmoc-chloride was used as the derivatizing reagent at a concentration of $8 \mathrm{mM}$ in acetonitrile. Borate buffer $(0.2 \mathrm{M})$ was prepared by dissolving $6.18 \mathrm{~g}$ boric acid in $425 \mathrm{~mL}$ water followed by $\mathrm{pH}$ adjustment to 8.5 using $\mathrm{NaOH}$ (10 M). A $300 \mathrm{mM}$ solution of amantadine was prepared in acetonitrile/water $(1: 1, \mathrm{v} / \mathrm{v})$ and was used as a scavenger for the unreacted Fmocchloride.

To $0.1 \mathrm{~mL}$ of rat plasma was added $50 \mu \mathrm{L}$ of IS and proteins precipitated with $200 \mu \mathrm{L}$ of cold acetonitrile followed by $1 \mathrm{~min}$ vortex-mixing and centrifugation for $3 \mathrm{~min}$ at $10,000 \mathrm{~g}$. $100 \mu \mathrm{L}$ of the supernatants were transferred into clean test tubes and $50 \mu \mathrm{L}$ of each borate buffer and a freshly prepared Fmoc-chloride solution were added. After 1 min vortex-mixing the samples were incubated in a water bath at $30^{\circ} \mathrm{C}$ for $30 \mathrm{~min}$. Subsequently, 50 $\mu \mathrm{L}$ of amantadine was added and samples were diluted with $1 \mathrm{~mL}$ acetonitrile/water (1:1) followed by injection of aliquots $(5 \mu \mathrm{L})$ into HPLC.

Three calibration curves were prepared on the same day to determine intra-day variability. The experiment was repeated on three different days to determine the inter-day variability. The accuracy was determined from \% error $=$ (mean observed concentration - added concentration) x100/ added concentration. The coefficient of variation $(\mathrm{CV} \%)$ was used to estimate the assay precision.

The percent recovery of GlcN, GVG, and IS from plasma was estimated from \% recovery = (compound peak height in plasma sample / compound peak height in water sample) x 100 .

In order to determine the short term stability, samples $(0.05,0.5,5$ and $20 \mu \mathrm{g} / \mathrm{mL})$ were derivatized and analyzed at $0,4,8$ and 24 h postderivatization. In order to measure the freeze and thaw stability, samples were prepared and kept in the freezer at $-20^{\circ} \mathrm{C}$ for $24 \mathrm{~h}$. The samples were removed from the freezer and allowed to thaw at room temperature followed by refreezing for another $24 \mathrm{~h}$. Aliquots of the samples were derivatized and analyzed after each freeze-thaw cycle. At the end of the stability experiments, the \% accuracy and $\mathrm{CV} \%$ were calculated.

\section{Bioavailability of ester derivatives}

Under oxygen/methoxyflurane anaesthesia, animals were cannulated in their right jugular vein as previously described (1). Briefly, a polyethylene cannula (Dow Corning Corp., Midland, MI, USA) tipped with $2 \mathrm{~cm}$ of silastic tubing (Becton Dickinson, Sparks, MD, USA) was inserted into their right jugular vein. Rats were allowed to recover overnight. Food was withdrawn from the animals $12 \mathrm{~h}$ before dosing. They were randomly assigned into four different groups ( $\mathrm{n}=8-11$ /group) and received either GlcN, or one of its ester prodrugs (GVG, FFG, or VGG) at the equivalent to $100 \mathrm{mg} / \mathrm{kg}$ of GlcN and serial blood samples were collected just before administration and at $0.25,0.5$, $0.75,1,1.5,2,3$, and $4 \mathrm{~h}$ post-dose. Samples were collected into heparinized tubes and plasma separated immediately by centrifugation. Total urine output was also collected over a period of 24 h. All samples were stored at $-20^{\circ} \mathrm{C}$ until analyzed.

\section{Fecal stability of GVG}

Fecal pellets were collected from healthy rats (one pellet from each rat; $\mathrm{n}=6$ ) and placed in $50 \mathrm{~mL}$ centrifuge tube, soften by addition of $25 \mathrm{~mL}$ deionised water, vortex-mixed for $10 \mathrm{~min}$ and centrifuged at $2500 \mathrm{rpm}$ for $8 \mathrm{~min}$. The fecal suspension $(1 \mathrm{~mL})$ was transferred into a $1.5 \mathrm{~mL}$ microcentrifuge tube and centrifuged at $8,300 \mathrm{~g}$ for $5 \mathrm{~min}$. The supernatant was immediately spiked with GVG (1.25 mg per each sample). The first set of samples was incubated at $37^{\circ} \mathrm{C}$ for $2 \mathrm{~h}$ to assess the effect of aerobic intestinal micro-flora. Air was removed from the tubes of the second set of samples $(n=6)$ by blowing nitrogen $\left(\mathrm{N}_{2}\right)$ into the test tubes. Thereafter, samples were incubated at $37^{\circ} \mathrm{C}$ for $2 \mathrm{~h}$ to assess the effect of anaerobic intestinal micro-flora. The third set of samples was placed in a $4^{\circ} \mathrm{C}$ refrigerator for $2 \mathrm{~h}$ in order to study the effect of the feces compositions on GVG stability. A GVG solution $(1.25 \mathrm{mg})$ with addition of no feces was set separately as a negative control. Control sample was incubated for $2 \mathrm{~h}$ at $37^{\circ} \mathrm{C}$. At the end of the experiment the amount of GVG was determined using HPLC. 


\section{Effect of inflammation on GVG bioavailability}

Animals were randomly divided into two groups: Inflamed or healthy control $(n=6)$. Adjuvant arthritis was induced in the inflamed animals by injecting $0.2 \mathrm{~mL}$ of Mycobacterium butyricum in squalene $(50 \mathrm{mg} / \mathrm{mL})$ in the tail base. Healthy rats were injected $0.2 \mathrm{~mL}$ of saline. On day 14, when the sign and symptoms of arthritis appear in the inflamed group (1), all animals were cannulated in their right jugular vein. After an overnight recovery period, a dose of GVG (equivalent to $100 \mathrm{mg} / \mathrm{kg}$ of GlcN base) was administered and serial blood samples were collected before dosing and at 0.25 , $0.5,0.75,1,1.5,2,3$, and $4 \mathrm{~h}$ post dosing. The total 0-24 $\mathrm{h}$ urine output was also collected for the determination of GVG and GlcN.

\section{GVG efficacy in prevention of adjuvant arthritis} Rats were randomly assigned into 6 groups $(n=6$ /group): Control-Healthy, Control-Inflamed, GlcN20, GVG-20, GVG-30, and GlcN-90. On day one, all inflamed rats were injected $0.2 \mathrm{~mL}$ of Mycobacterium butyricum in squalene $(50 \mathrm{mg} / \mathrm{mL})$. Control-Healthy animals were injected $0.2 \mathrm{~mL}$ of normal saline. GlcN or GVG administration was commenced on the first day of experiment as a preventive measure. In this study we chose 2 daily GlcN equivalent dosage regimens: First, $30 \mathrm{mg} / \mathrm{kg}$ GVG vs $90 \mathrm{mg} / \mathrm{kg}$ GlcN to confirm efficacy at doses higher the minimum effective GlcN dose (40 $\mathrm{mg} / \mathrm{kg}$, (13)), and second, $20 \mathrm{mg} / \mathrm{kg} \mathrm{GVG}$ versus 20 $\mathrm{mg}$ GlcN, a regimen below the minimum effective GlcN dose.

During the experiment, body weight gain and paw thickness (using a caliper with a sensitivity of $25 \mu \mathrm{m}$, Mitutoyo Canada Inc., Toronto, ON) were measured daily. On the last day of experiment, serum nitrite was measured using a previously described method (14). Nitrite standard curves were linear over the concentration range of $3-200 \mu \mathrm{M}$ $\left(\mathrm{r}^{2} \geq 0.99\right.$, coefficient of variation $\left.\leq 10 \%\right)$.

On day 18 after injection of the adjuvant, arthritis index was calculated according to a previously described method (15). A maximum score of 14 could be assigned to each animal as followed: On a 0-4 basis hind paws were scored where zero was no virtual sign of arthritis, one was involvement of a single joint, two was involvement of more than one joint and/or ankle, three was involvement of several joints and ankle with moderate swelling, and four was involvement of several joints and ankle with severe swelling. Each forepaw was scored on a $0-3$ basis scoring system where 0 was involvement of none of the joints, one was involvement of single joint, two was involvement of more than one joint and/or wrist, and three was involvement of wrist and joints with moderate-to-severe swelling.

\section{Data treatment and statistical analysis}

The non-compartmental approach was used to calculate pharmacokinetic parameters. The area under the plasma concentration-time curve (AUC) $0-4 \mathrm{~h}$ post-dose was calculated using the trapezoidal method. The peak concentration $\left(\mathrm{C}_{\max }\right)$ and the time of its attainment $\left(t_{\max }\right)$ were the experimentally observed values. The percent urinary excretion was estimated from (total excreted in urine in $24 \mathrm{~h} /$ dose) X 100.

Data are presented as mean \pm SD. Differences between two means were assessed using the Student's t-test (Table 2). For more than two means, we used the one-way ANOVA followed by Bonferroni post-test. All statistical analyses were carried out using Prism software (GraphPad Software Inc., San Diego, CA, USA) at $p<0.05$.

\section{RESULTS}

\section{Simultaneous HPLC assay for GIcN and GVG}

The HPLC peaks for Fmoc derivatized GlcN and mannosamine (IS) appeared as pairs of anomers as has been previously reported (8). The anomers resolved at 11.6 and $14.1 \mathrm{~min}$ for the IS and 12.7 and $15.5 \mathrm{~min}$ for GlcN (Figure 1). The second peak of each compound was used in order to quantify the samples. GVG resolved as a single peak at a retention time of $17.7 \mathrm{~min}$. No interfering peak was observed in the blank rat plasma or urine samples.

The assay was linear over the range of $0.05-20$ $\mu \mathrm{g} / \mathrm{mL}$ for both $\mathrm{GVG}$ and $\mathrm{GlcN}$ in rat plasma $\left(r^{2} \geq 0.99\right)$. The lowest limit of quantification was set at $50 \mathrm{ng} / \mathrm{mL}$ for both compounds. The peak height measurement approach provided lower variability in response than that of the peak area, hence, the former was used throughout. The inter- and intraday variations were less than $10 \%$ for all concentrations and the accuracy ranged from -1.4 to $2.4 \%$ for GlcN and -6 to $8 \%$ for GVG. 


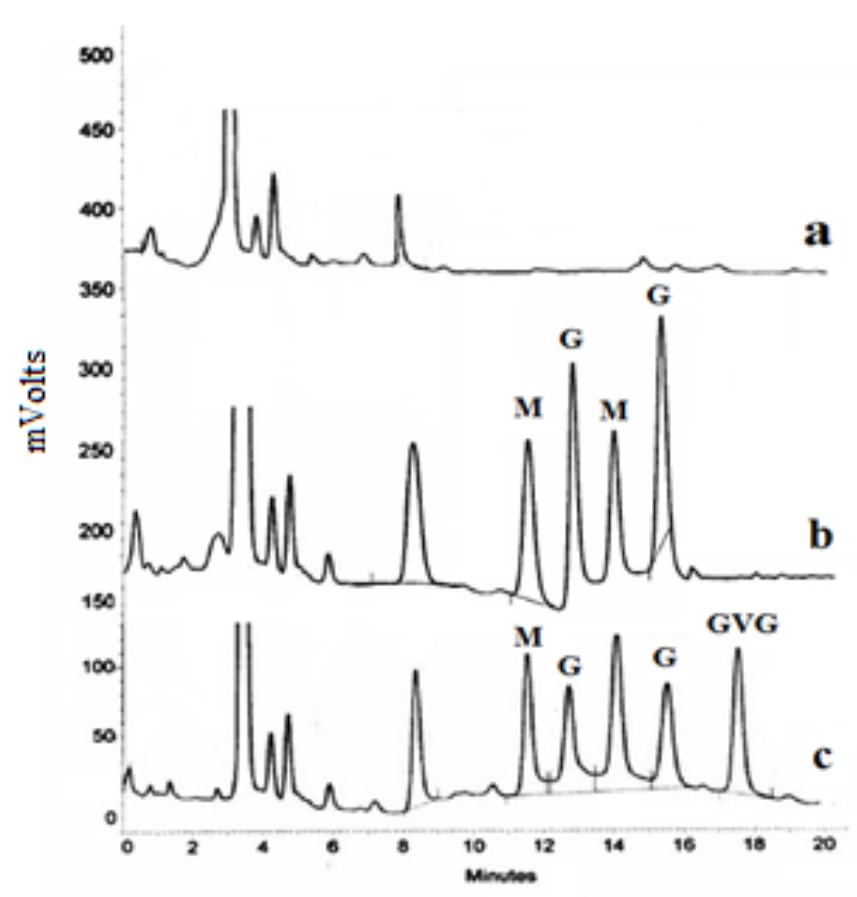

Figure 1. HPLC chromatograms of (a) blank rat plasma, (b) rat plasma at t=4 h post-dosing (GVG $100 \mathrm{mg} / \mathrm{kg})$; only GlcN peaks appears in plasma after GVG administration, and (c) blank rat plasma spiked with $1 \mu \mathrm{g} / \mathrm{mL}$ GlcN and GVG. Keys: M, mannosamine (IS); G, GlcN; GVG, Gly-Val-COO-GlcN

The percent recovery of the IS from rat plasma was $96.1 \pm 3$. The $\%$ recovery of GVG was $99 \pm 5.3$, $98.2 \pm 5.1,97 \pm 3.7,95.9 \pm 9.2$, and $94.2 \pm 7.3$ for the $0.05,0.5,5,10$, and $20 \mu \mathrm{g} / \mathrm{mL}$ samples in rat plasma, respectively. Percent recovery of GlcN from rat plasma appeared to be $105 \pm 6.1,97.8 \pm 7$, $103.2 \pm 4.3,103.3 \pm 2.2$, and $99.8 \pm 6.2 \%$ from the $0.05, \quad 0.5, \quad 5, \quad 10$, and $20 \mu \mathrm{g} / \mathrm{mL}$ samples, respectively.

Derivatized GVG and GlcN were stable in all plasma samples during the analysis process and 24 $\mathrm{h}$ after derivatization; stability was $>91 \%$ for all samples. GVG and GlcN were also appeared to be stable in rat plasma after three freeze and thaw cycles.

\section{Bioavailability of GlcN versus its ester derivatives}

Figure 2 depicts the mean plasma concentration of GlcN after administration of equivalent doses (100 $\mathrm{mg} / \mathrm{kg}$ of GlcN base) of GlcN or its ester derivatives. All compounds were rapidly absorbed $\left(\mathrm{t}_{\max } \leq 0.5 \mathrm{~h}\right)$. However, among all tested compounds, only GVG showed a significantly higher oral bioavailability as compared to GlcN
(Table 1). FFG and VGG only showed an insignificant trend toward increased bioavailability as compared to GlcN. GVG also exhibited a significant increase in percent of dose excreted in the urine as compared to the other tested compounds. No significant differences were

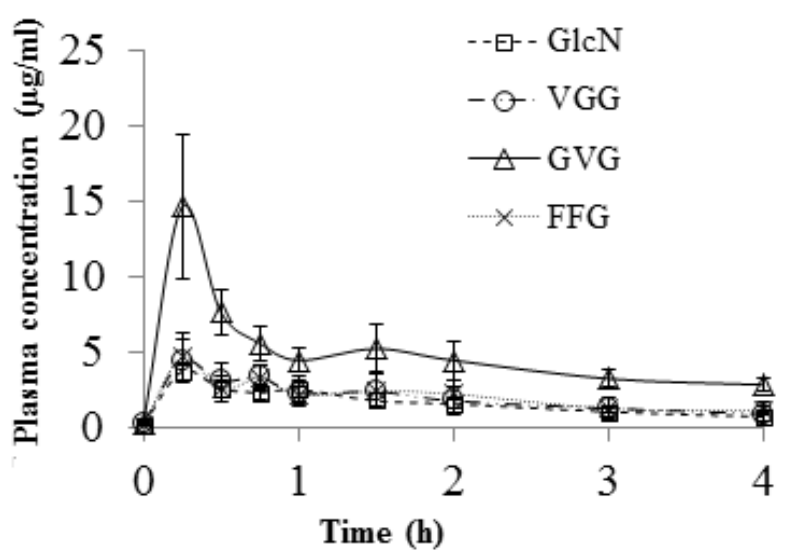

Figure 2. Mean GlcN plasma concentration ( \pm SD) versus time profile following oral administration of GlcN or the ester derivatives of GlcN to rats $(n=8-$ $11 /$ group). All doses were equivalent to $100 \mathrm{mg} / \mathrm{kg}$ of GlcN base. 
Table 1. Bioavailability parameters of GlcN and its ester derivatives

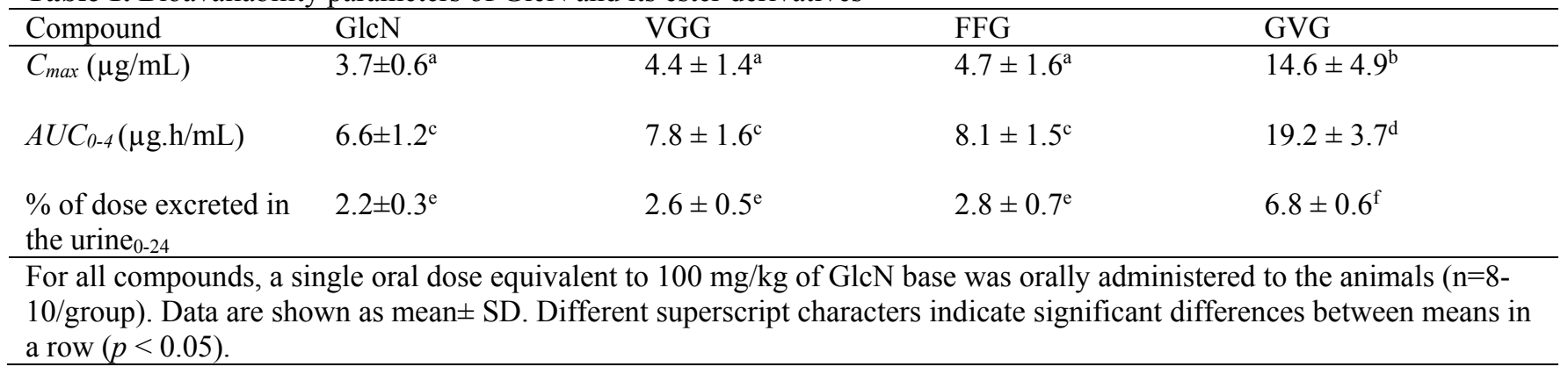

noticed among GlcN, FFG, and VGG in any of the calculated pharmacokinetic parameters.

No intact GVG was detected in plasma or urine after its oral administration.

\section{Fecal stability of GVG}

Figure 3 depicts the percent remaining of GVG after $2 \mathrm{~h}$ incubation under control (no feces), aerobic, and anaerobic conditions at 37 and $4^{\circ} \mathrm{C}$. Incubation of GVG with rat faces resulted in almost $33 \%$ loss in all samples. There were no significant differences between the amounts of GVG that remained at the end of the experiment under aerobic, anaerobic, or $4^{\circ} \mathrm{C}$ conditions. At the end of the experiment, approximately $97 \%$ of GVG remained intact in the control samples.

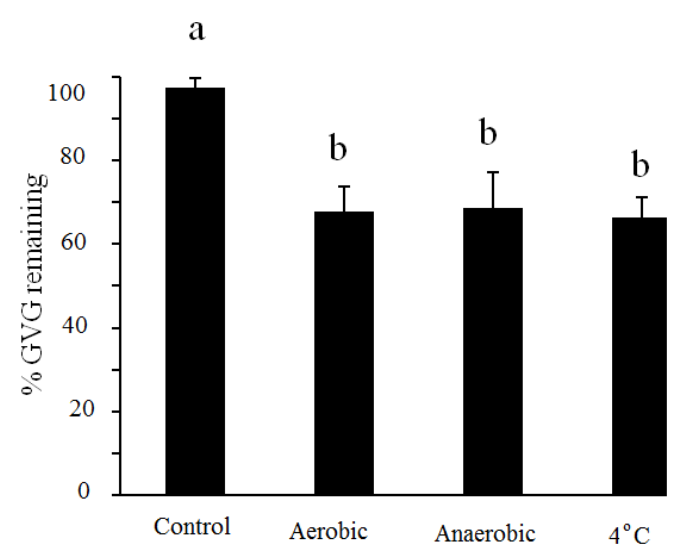

Figure 3. The average percent GVG remaining after $2 \mathrm{~h}$ incubation at $37^{\circ} \mathrm{C}$ (control, aerobic and anaerobic) or at $4^{\circ} \mathrm{C}$. Error bars represent standard deviation. The same character indicates no significant difference between means at $p<0.05 ; \mathrm{n}=6 /$ group.

\section{Effect of inflammation on GVG bioavailability} Inflammation did not significantly alter the oral bioavailability of a single oral dose of GVG (100 $\mathrm{mg} / \mathrm{kg}$ of GlcN base) as no significant differences were noticed between the groups in $\mathrm{C}_{\max }, \mathrm{AUC}_{0-4}$ and the percent of dose excreted in the urine in $24 \mathrm{~h}$ (Figure 4 and Table 2).

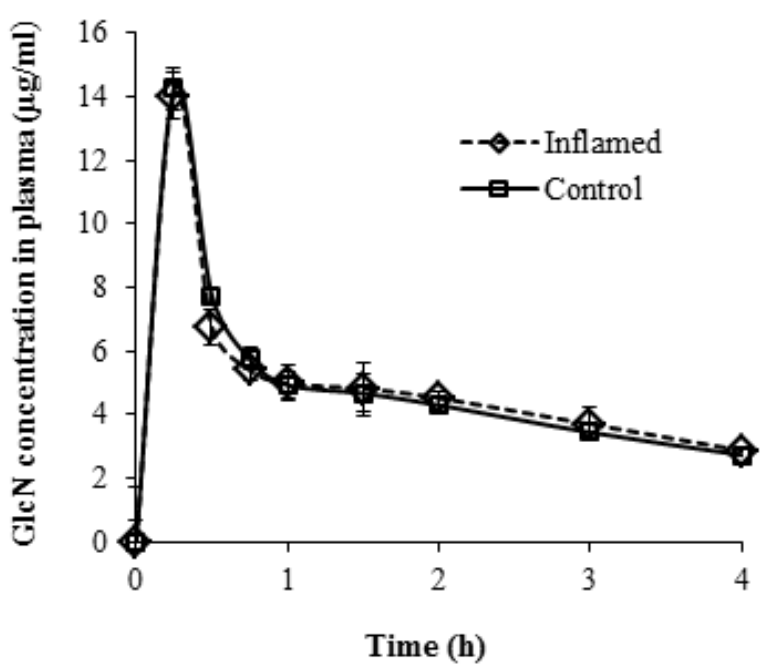

Figure 4. Mean ( $n=6 /$ group) plasma concentration $( \pm \mathrm{SD})$ versus time profiles of glucosamine in control and inflamed rats following oral administration of a single dose equivalent to $100 \mathrm{mg} / \mathrm{kg} \mathrm{GlcN}$ base.

\section{GVG efficacy in prevention of adjuvant arthritis} Adjuvant arthritis emerged in the rats (ControlInflamed) that received Mycobacterium butyricum but were not treated with either GlcN or GVG. They exhibited significant increase in paw thickness (Figure 5) as compared to the ControlHealthy animals and, at the end of the experiment (day 18) had arthritis index of $9.1 \pm 0.8$. No sign of arthritis was observed in Control-Healthy group as well as in the rats that were treated with 90 $\mathrm{mg} / \mathrm{kg} /$ day GlcN (GlcN-90) or 20 and 30 $\mathrm{mg} / \mathrm{kg} /$ day GVG. Administration of $20 \mathrm{mg} / \mathrm{kg} /$ day GlcN (GlcN-20) did not prevent adjuvant arthritis but the severity of the disease was significantly less than those observed for Control-Inflamed (arthritis index, $4.1 \pm 1.1)$. 
Table 2. Bioavailability parameters of GVG in control and inflamed rats ( $\mathrm{n}=6$ /group)

\begin{tabular}{lcc}
\hline \multicolumn{1}{c}{ Group } & Inflamed & Control \\
\hline$C_{\max }(\mu \mathrm{g} / \mathrm{mL})$ & $14.01 \pm 1.7$ & $14.25 \pm 0.6$ \\
$A U C_{0-4}(\mu \mathrm{g} . \mathrm{h} / \mathrm{mL})$ & $19.1 \pm 1.1$ & $18.97 \pm 0.5$ \\
$\%$ of dose excreted in the urine ${ }_{0-24}$ & $7.1 \pm 0.6$ & $6.9 \pm 0.5$ \\
\hline
\end{tabular}

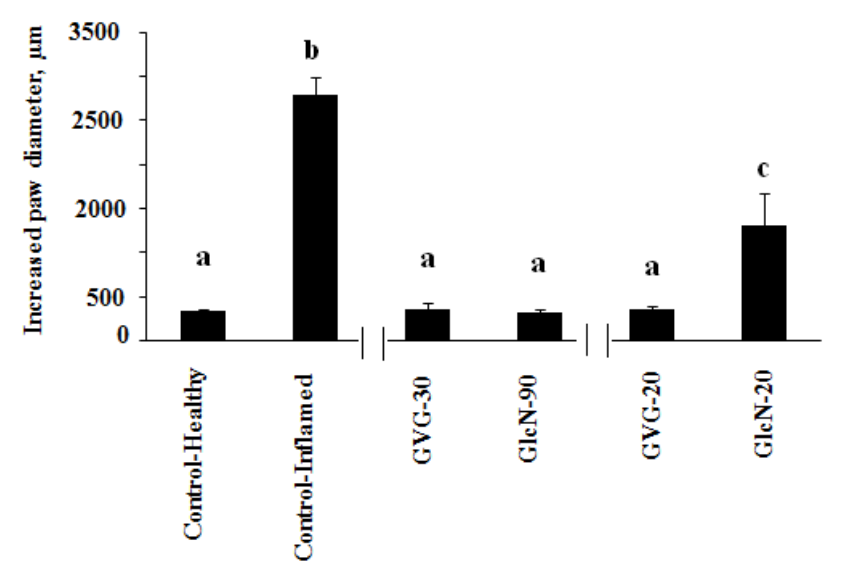

Figure 5. Average increase in paw thickness at the end of the experiment. Error bars represent standard deviation. The same character indicates no significant difference between means at $p<0.05 ; \mathrm{n}=6$ /group.

The effect of the treat ments on the weight gain (Figure 6) and serum nitrite levels (Figure 7) were similar to those observed for adjuvant arthritis.

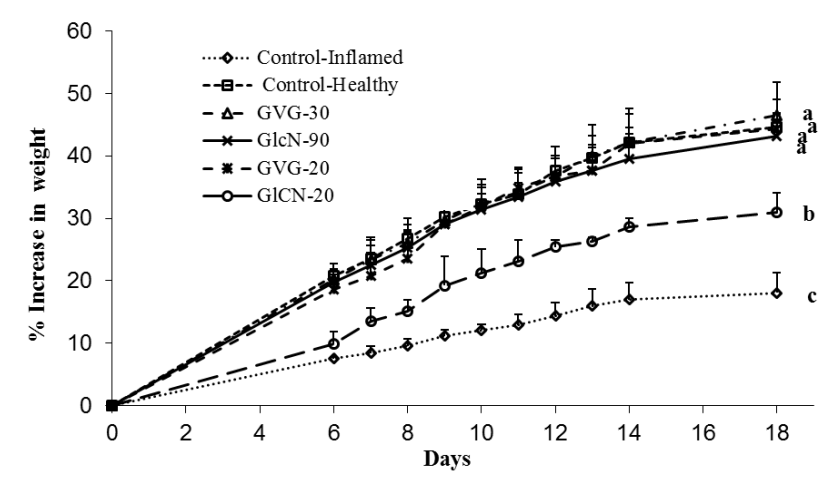

Figure 6. The average percent weight gain $(+\mathrm{SD})$ from baseline. Error bars represent standard deviation. The same character indicates no significant difference between means at $p<0.05 ; \mathrm{n}=6$ /group.

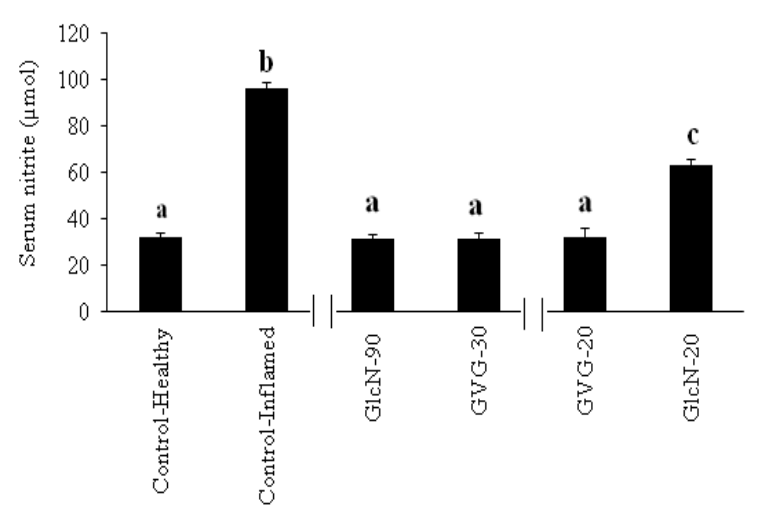

Figure 7. The effect of inflammation and prevention with GlcN or GVG on serum nitrite concentration in inflamed rats ( $n=6$ /group); error bars represent standard deviation. The same character indicates no significant difference between means at $p<0.05$.

\section{DISCUSSION}

\section{Assay of GVG and GIcN}

We developed a sensitive HPLC assay for simultaneous measurement of GlcN and its ester derivative, GVG, based on fluorescence detection. As neither GlcN nor GVG contain fluorophore groups, we derivatized them to Fmoc-GlcN and Fmoc-GVG. Fmoc-Cl has been used since 1980s for HPLC analysis of amino acids (7). It rapidly and almost completely reacts with primary and secondary amines under alkaline conditions. The solvent gradient program and the addition of $0.1 \%$ acetic acid to the mobile phase resulted in sharp and resolved peaks (Figure 1). Most of the plasma interfering peaks eluted before the appearance of the peaks of interest. The derivatization method yields two equal sized but completely resolved anomers (stereoisomers of cyclic sugars that differ in their configuration at the anomeric carbon) peaks (8). The excessive amount of the derivatizing agent was removed by adding amantadine to form a hydrophobic complex that eluted at the end of the run time and before the next injection. To prevent 
pump pressure build-up, the injection volume was set at $5 \mu \mathrm{L}$.

The method was linear over the examined range with suitable sensitivity and acceptable accuracy and reproducibility.

\section{Bioavailability}

All of the three examined di-peptide GlcN esters demonstrated trends toward a greater oral bioavailability than the parent compound but the change reached statistical significance only for GVG. This is in agreement with the previously reported seven-fold greater than GlcN permeability across the everted rat gut for GVG (6). The enhanced permeability was attributed to the involvement of PepTl since incubation in the presence of PepT1 inhibitor (Gly-Sar) reduced the process to the level of that observed for GlcN (6). Our observation may be proven useful in predicting oral absorption of these compounds following administration to humans, since rats appear to be suitable predictors of drug absorption by humans (16). The high permeability of GVG is due, likely, to the recognition of the conjugate by the PepT1, as a specific PepT1 substrate; Gly-Sar completely inhibited transport of GVG (6). In general, the 3dimensional conformation properties of a substrate govern whether a substrate has affinity for PepT1 (17-19). GVG seemed to have the appropriate stereochemical and conformational features. There are several other examples in which researchers have attempted to improve oral absorption of various poorly absorbed drugs via attachment to amino acids or dipeptides in order to resemble PepT1 substrates, mostly through ester bonds (2023). Similar to GVG, the glycine-valine ester prodrug of acyclovir appeared to be more efficiently absorbed across the intestinal mucosa as compared to other ester derivatives (20).

GVG has been shown to be relatively stable in the gut; it readily crosses the gut wall and rapidly yields the parent drug upon incubation with liver homogenates (6). Although it has been shown that GVG remains intact during movement from mucosal toward serosal side in the rat small intestine (6), we were not able to detect any intact GVG in the systemic circulation. In other words GVG seems to be transported into the portal vein and then cleaved into GlcN and the di-peptide moiety either in the liver.
In general, the activation of the prodrug such as GVG after its in vivo hydrolysis, is not expected to raise toxicity concerns since the activation byproducts are a dipeptide and/or amino acids and the parent drug $(20,24)$.

The low bioavailability of GlcN is attributed to, at least, two mechanisms, the inefficiency of the involved transporters (i.e., GLUT-2) and the loss due to the uptake by the gut microflora (9). It has been shown that incubation of $1.25 \mathrm{mg}$ GlcN with the rat feces results in $95 \%$ loss. For GVG only $33 \%$ loss was detected over a $2 \mathrm{~h}$ incubation period (Figure 3). The mechanism of this loss is unclear as it occurs under various conditions (aerobic, anaerobic and/or $4^{\circ} \mathrm{C}$ ) that may put the mere involvement of microflora into question. Nevertheless, the observed fecal stability of GVG as compared with GlcN may contribute to the greater bioavailability of the latter.

\section{Efficacy}

The rat model of AA mimics human rheumatoid arthritis which has been vastly used for decades to assess the efficacy of antirheumatic agents (10). We used this model to compare the antiinflammatory effects of GVG with GlcN. Assuming a three-fold greater bioavailability of GVG as compared with GlcN, we first compared $30 \mathrm{mg} / \mathrm{kg} /$ day of the former with $90 \mathrm{mg} / \mathrm{kg} /$ day (GlcN equivalent) of the latter to assess the efficacy of the same GlcN exposure following administration of the two different compounds. We noticed that the treatments were equally effective in preventing adjuvant arthritis (Figure 5), reduced body weight gain (Figure 6) and increased serum nitrite level (Figure 7). Considering the fact that the oral administration of GVG yields only GlcN in the systemic circulation, one can suggest that the greater potency of GVG is due to the corresponding higher GlcN bioavailability after administration of the prodrug as compared to the parent compound. To further confirm the observation, we compared the two compounds following daily treatment with $20 \mathrm{mg} / \mathrm{kg}$ /day (GlcN equivalent), which was known to be below the GlcN effective dose range (13). The GVG and not GlcN regimen was effective in completely preventing the experimental arthritis. This further confirms that, at the dosage level that GlcN is not fully effective, GVG prevents the emergence of adjuvant arthritis. 


\section{Effect of inflammation of GVG pharmacokinetics}

Inflammation may alter clearance of drugs that are efficiently metabolized drugs (25) and also influence the expression of some transporters (11). Inflammation did not result in such an effect on GVG since the plasma GlcN concentration-time curves following GVG administration under healthy and inflammatory conditions were almost superimposable (Figure 4). This suggest a lack of effect of inflammation on the activation of the prodrug and/or clearance of the active compound (glucosamine) as has been reported earlier (1). Similarly, according to Radeva et al (2007), experimentally-induced rat colitis, another inflammatory condition, does not alter the bioavailability of cephalexin or valacyclovir, two known PepTl substrates despite certain modifications in the transporter mRNA expression (12).

\section{CONCLUSION}

A novel dipeptide ester of GlcN, GVG, is found to have an increased bioavailability assessed using a novel HPLC method. GVG exhibited increased anti-inflammatory property through a greater GlcN delivery into the systemic circulation. The pharmacokinetics of GlcN delivered through GVG is not influenced by adjuvant arthritis. If the present nonclinical data are extrapolated to humans, GVG can be considered as an alternative to other antiinflammatory agent that have gastrointestinal (26), renal (27) and/or cardiovascular (28) side effects.

\section{REFERENCES}

1. Gilzad-Kohan MH, Jamali F. Glucosamine and adjuvant arthritis: a pharmacokinetic and pharmacodynamic study. Eur J Pharm Sci 2012 Sep 29;47(2):387-393.

2. Hua J, Suguro S, Hirano S, Sakamoto K, Nagaoka I. Preventive actions of a high dose of glucosamine on adjuvant arthritis in rats. Inflamm Res 2005 Mar;54(3):127-132.

3. Hwang SY, Shin JH, Hwang JS, Kim SY, Shin JA, Oh ES, et al. Glucosamine exerts a neuroprotective effect via suppression of inflammation in rat brain ischemia/reperfusion injury. Glia 2010 Nov 15;58(15):1881-1892.
4. Aghazadeh-Habashi A, Jamali F. The glucosamine controversy; a pharmacokinetic issue. J Pharm Pharm Sci 2011;14(2):264-273.

5. Ragle RL, Sawitzke AD. Nutraceuticals in the management of osteoarthritis : a critical review. Drugs Aging 2012 Sep;29(9):717-731.

6. Gilzad-Kohan M, Kaur K, Jamali F. From Nutraceutical to Pharmaceutical: A Novel Peptide Pro-drug of Glucosamine with Increased Bioavailability. J Pharm Pharm Sci. 2011, 14 (3): $157 \mathrm{~s}$.

7. Einarsson S, Josefsson B, Lagerkvist S. Determination of amino acids with 9fluorenylmethyl chloroformate and reversed-phase high-performance liquid chromatography. Journal of Chromatography A 1983 12/30;282(0):609-618.

8. Ibrahim A, Jamali F, Improved Sensitive High Performance Liquid Chromatography Assay for Glucosamine in Human and Rat Biological Samples with Fluorescence Detection. Journal of Pharmacy \& Pharmaceutical Sciences 2010;13(2):128.

9. Ibrahim A, Gilzad-Kohan MH, Aghazadeh-Habashi A, Jamali F. Absorption and bioavailability of glucosamine in the rat. J Pharm Sci 2012 Jul;101(7):2574-2583.

10. Rosenthale ME, Capetola RJ. Adjuvant arthritis: immunopathological and hyperalgesic features. Fed Proc 1982 Jul;41(9):2577-2582.

11. Cressman A, Petrovic V, Piquette-Miller M. Inflammation-mediated changes in drug transporter expression/activity: implications fortherapeutic drug response.Expert Rev Clin Pharmacol. 2012 Jan;5(1):69-89.. 2012.

12. Radeva G, Buyse M, Hindlet P, Beaufils B, Walker F, Bado A, et al. Regulation of the oligopeptide transporter, PEPT-1, in DSS-induced rat colitis.Dig Dis Sci. 2007 Jul;52(7):1653-61. Epub 2007 Mar 20.. 2007.

13. Agahzadeh-Habashi A, Gilzad-Kohan M, Jamali F. Dose Escalation Study of Glucosamine to Treat Adjuvant Arthritis in the Rat. J Pharm Pharm Sci 2011; 14 (3): 149s-150s.

14. Grisham MB, Johnson GG, Gautreaux MD, Berg RD. Measurement of nitrate and nitrite in extracellular fluids: a window to systemic nitric oxide metabolism. Methods 1995;7(1):84-90.

15. Piquette-Miller M, Jamali F. Influence of severity of inflammation on the disposition kinetics of propranolol enantiomers in ketoprofen-treated and untreated adjuvant arthritis. Drug Metab Dispos 1995 Feb;23(2):240-245.

16. Cao X, Gibbs S, Fang L, Miller H, Landowski C, Shin $\mathrm{H}$, et al. Why is it Challenging to Predict Intestinal Drug Absorption and Oral Bioavailability in Human Using Rat Model. Pharmaceutical Research 2006;23(8):1675-1686. 
17. Bailey PD, Boyd CA, Bronk JR, Collier ID, Meredith D, Morgan KM, et al. How to Make Drugs Orally Active: A Substrate Template for Peptide Transporter PepT1. Angew Chem Int Ed Engl 2000 Feb;39(3):505-508.

18. Bailey PD, Boyd CA, Collier ID, George JP, Kellett GL, Meredith D, et al. Affinity prediction for substrates of the peptide transporter PepT1. Chem Commun (Camb) 2006 Jan 21;(3)(3):323-325.

19. Rubio-Aliaga I, Daniel H. Peptide transporters and their roles in physiological processes and drug disposition. Xenobiotica 2008 Jul;38(7-8):10221042.

20. Anand BS, Katragadda S, Mitra AK. Pharmacokinetics of novel dipeptide ester prodrugs of acyclovir after oral administration: intestinal absorption and liver metabolism. J Pharmacol Exp Ther 2004 Nov;311(2):659-667.

21. Weller S, Blum MR, Doucette M, Burnette T, Cederberg DM, de Miranda $P$, et al. Pharmacokinetics of the acyclovir pro-drug valaciclovir after escalating single- and multipledose administration to normal volunteers. Clin Pharmacol Ther 1993 Dec;54(6):595-605.

22. Szczech GM. Preclinical development of antiviral drugs. Clin Infect Dis 1996 Feb;22(2):355-360.
23. Purifoy DJ, Beauchamp LM, de Miranda P, Ertl P, Lacey S, Roberts G, et al. Review of research leading to new anti-herpesvirus agents in clinical development: valaciclovir hydrochloride $(256 \mathrm{U}$, the L-valyl ester of acyclovir) and $882 \mathrm{C}$, a specific agent for varicella zoster virus. J Med Virol 1993;Suppl 1:139-145.

24. Krylov IS, Kashemirov BA, Hilfinger JM, McKenna CE. Evolution of an amino Acid based prodrug approach: stay tuned. Mol Pharm 2013 Feb 4;10(2):445-458.

25. Guirguis MS, Jamali F. Disease-drug interaction: reduced response to propranolol despite increased concentration in the rat with inflammation. $\mathrm{J}$ pharm sci 2003; 92:1077-1084

26. Davies NM, Jamali F. Pharmacological protection of NSAID-induced intestinal permeability in the rat: effect of tempo and metronidazole as potential free radical scavengers. Human \& experimental toxicology 1997;16), 345-349.

27. Harirforoosh S, Jamali F. Renal adverse effects of nonsteroidal anti-inflammatory drugs. Expert opinion on drug safety 2009; 8, 669-681.

28. Davies NM,Jamali F. COX-2 selective inhibitors cardiac toxicity: getting to the heart of the matter. J Pharm Pharm Sci 2004; 7:332-336. 